\title{
SIMS Detector on FIB/SEM DualBeam Microscopes for Material Science Applications
}

Chengge Jiao ${ }^{1}, \mathrm{Xu} \mathrm{Xu}^{2}$, Timothy Burnett ${ }^{2}$, Mikhail Dutka ${ }^{1}$, David Wall ${ }^{1}$ and Brandon van Leer ${ }^{3}$

${ }^{1}$ ThermoFisher Scientific, Eindhoven, Noord-Brabant, Netherlands, ${ }^{2}$ The University of Manchester, Manchester, England, United Kingdom, ${ }^{3}$ ThermoFisher Scientific, Hillsboro, Oregon, United States

For material science samples, micro-chemical measurement by energy dispersive $\mathrm{x}$-ray microanalysis (EDX) with a DualBeam FIB/SEM microscope is a standard technique. However, this technique for detection of hydrogen is impossible, and the detection of lithium requires well defined measurement conditions and is very challenging [1]. Secondary Ion Mass Spectrometry (SIMS) is a materials chemical analysis technique relies on collection, separation of ions according to their mass-to-charge ratio $(\mathrm{m} / \mathrm{z})$ from detected secondary ions. The secondary ions are one of emitted signals from solid material surfaces by primary ion beam bombardment in a FIB/SEM DualBeam microscope.

Add-on a SIMS detector to a DualBeam FIB/SEM microscope to collect secondary ions from a FIB/SEM microscope allows focused ion beam to be used not only for major functions of sample preparations and nanofabrications, but also for collecting sample chemical information by ion beam. The integrated SIMS to a FIB/SEM DualBeam microscope gives a far better depth $(\mathrm{z})$ resolution for chemical analysis because ion beam interaction volume is in general orders of magnitude smaller than a common SEM/EDX technique at the same beam energy. FIB/SEM/SIMS also delivers a better lateral $(\mathrm{x}, \mathrm{y})$ resolution comparing to dedicated SIMS systems because the focused ion beam has a smaller probe size.

In this paper, we give an overview of an integrated ToF SIMS to FIB/SEM DualBeam for materials science applications, focusing on SIMS for nano-analysis and to detect light, low concentration elements. First, we give a short overview and discussion of the pros and cons of current mass analyzers FIB/SEM microscopes. Results presented in this paper were mainly acquired from a gallium FIB DualBeam with a compact ToF SIMS detector added on to it [2,3], although plasma FIB Xe+ beam is also mentioned.

High resolution nano SIMS results from a sample of mixed organic/inorganic nanoparticles and a sample with a low content of rubidium segregated in $\mathrm{Cu}$ (In,Ga)Se2 (CIGS) grain boundaries [4] are presented. SIMS signal enhancement in post-processing is described. SIMS images from nanoparticles and from a sample of WC with a binder material cobalt proved that a Gallium FIB/ToF SIMS is capable of doing nano SIMS analysis down to about $20-30 \mathrm{~nm}$.

ToF SIMS results clearly show a distinguished advantage for the measurement of light element, in this case, lithium in an Al-Li alloy sample. By using FIB TEM specimen preparation as a link, we have tried to bridge microscopic chemical analysis techniques between FIB SEM SIMS measurements and TEM STEM EDS analysis in Al-Li sample. The TEM specimen was prepared as Gallium-free with a Plasma $\mathrm{Xe}^{+}$FIB from the same location where SIMS was performed. Sample correlations between different microscopes relies on SEM BSE images.

In conclusion, integrated ToF SIMS in a DualBeam SEM/FIB system allows the ion beam to be used not only for milling and deposition but also as a beam for chemical analysis. Light mass detection capability and good detection efficiencies makes the SIMS technique powerful for materials science research. FIB/SEM/SIMS gives better correlation between electron imaging techniques comparing to dedicated SIMS. Combining SIMS measurements in FIB/SEM microscope; linked with site-specific planar view TEM specimen preparation, and EDX analysis by HR TEM/STEM, provides near a complete sample chemical information for materials science research. 

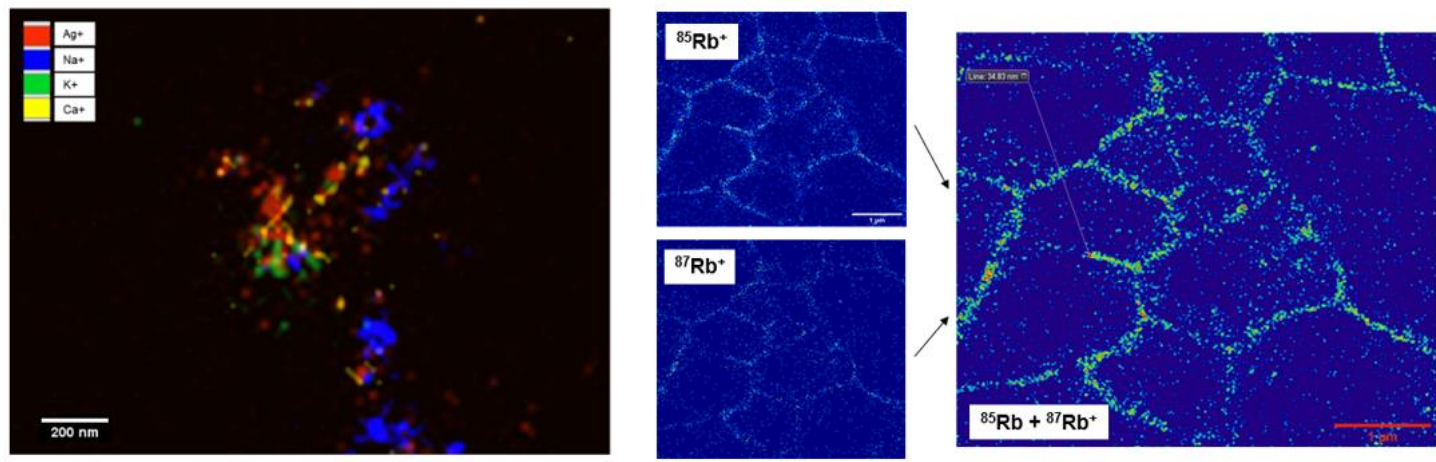

Figure 1. High resolution ToF SIMS images. (a) a color mixed ToF SIMS image of $107 \mathrm{Ag}+$ and $109 \mathrm{Ag}+$, $23 \mathrm{Na}+, 39 \mathrm{~K}+, 40 \mathrm{Ca}+$ from nanoparticles on silicon surface. (b) a low content heavy alkali element rubidium segregation in $\mathrm{Cu}(\mathrm{In}, \mathrm{Ga}) \mathrm{Se} 2$ solar cell sample.

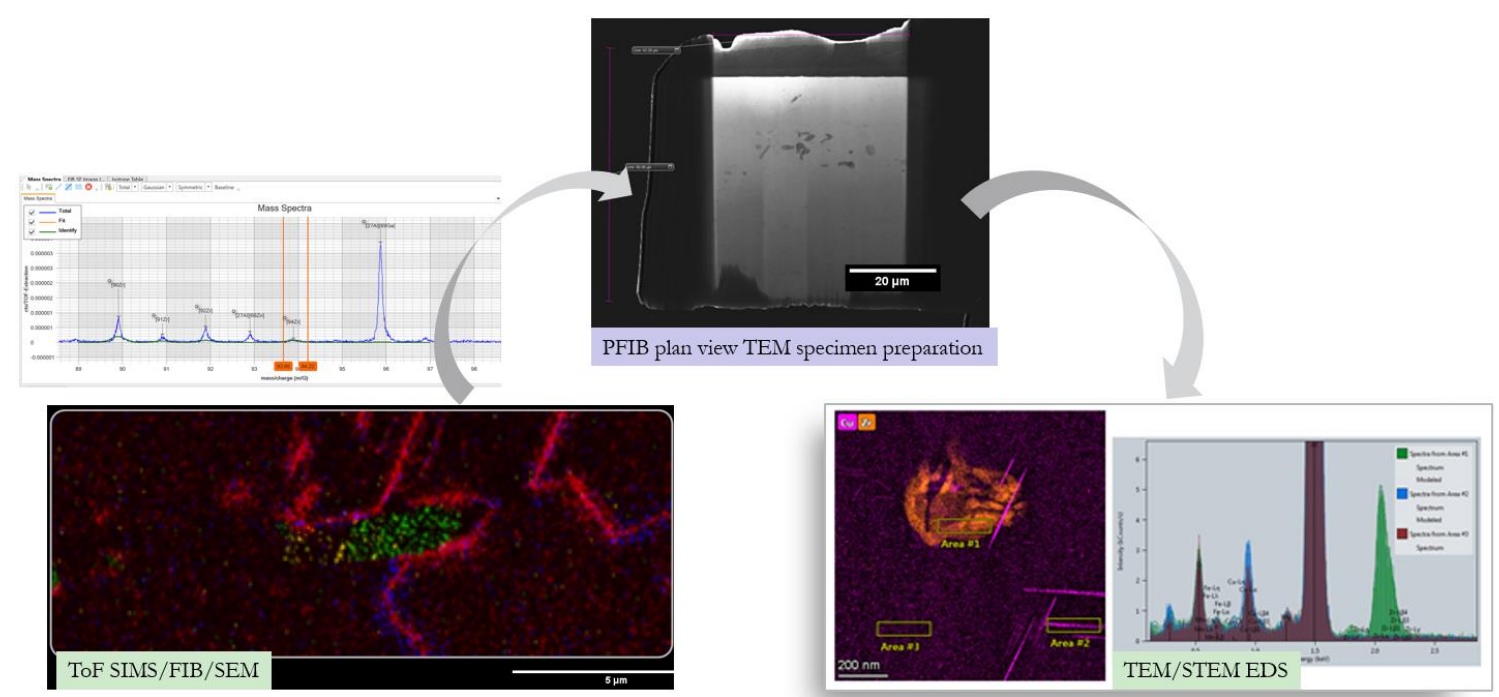

Figure 2. A combined Al-Li alloy sample chemical micro-/nano- measurements by ToF SIMS/FIB/SEM and STEM EDS by a TEM. The bridge of the analysis is a PFIB prepared gallium-free TEM specimen. The SIMS image shows lithium segregations at grain boundaries. Zirconium isotopes mass peaks are shown in the mass spectrum but was unable to form a SIMS image because of small isolated particles. TEM STEM EDS proved that the Zirconium particles in the alloy also proved that the Lithium-rich phase in needle shape.

\section{References}

[1] Pierre Hovington et al., Scanning VOL. 38, (2016), p.571-578

[2] Lex Pillatsch, Fredrik Ostlund, Johann Michler, Progress in Crystal Growth and Characterization of Materials, December 2018, https://doi.org/10.1016/j.pcrysgrow.2018.10.001

[3] Chengge Jiao, Lex Pillatsch, Johannes Mulders, David Wall, Microscopy and Microanalysis 25(S2):876877, DOI: $10.1017 /$ S1431927619005117

[4] Tom Wirtz, Olivier De Castro, Jean-Nicolas Audinot, and Patrick Philipp, Annual Review of Analytical Chemistry, 2019,12: 523-43, https://doi.org/10.1146/annurev-anchem-061318-115457 